\title{
THE EXTENDED RADIO STRUCTURE OF QUASARS
}

\author{
Frazer N. Owen \\ National Radio Astronomy Observatory \\ P.O. Box O \\ Socorro, NM 87801 \\ USA
}

\begin{abstract}
Modern radio maps usually allow quasars to be recognized from their radio morphology alone. Most have strong central components, double lobed outer structure and one-sided jets connecting the inner and outer structures. The physics of the sources is poorly understood. The observed bending of the jets, the high minimum pressures observed, and the required energy supply to the lobes are major problems. However, the outstanding problem regarding the extended structure is whether or not this morphology is produced by special relativistic effects or the intrinsic activity level and physics of the radio sources.
\end{abstract}

\section{Introduction, Basic Properties}

A decade ago our knowledge of the extended structure of quasars was limited to the fact that they generally resembled radio galaxies. We knew that resolved, high luminosity radio sources, whether identified with galaxies or quasars, most often were double or triple sources centered on the optical identification. Today with a new generation of radio interferometers and new image processing techniques we have a much improved picture and one can, in most cases recognize the difference between a radio galaxy and a quasar from the radio map alone. The two outstanding differences between radio galaxies and quasars with strong radio emission are 1) the strength of the radio jet and 2) the strength of the core component. Starting with the discovery of the radio jet in 4C 32.69 (Potash and Wardle, 1980), it has become increasingly clear that many, probably most or all, radio quasars possess a one-sided radio jet connecting the optical quasar with the extended lobe emission. Often these jets are knotty and show pronounced bends and wiggles, sometimes of $90^{\circ}$ or more. Roughly 100 quasar jets have been found to date and none are known which are clearly two-sided. In figure 1 several examples of quasars with the characteristic one-sided structure are shown.

Second, almost all radio quasars with integrated flux densities greater than, say, $1 \mathrm{Jy}$ at $1 \mathrm{GHz}$, have central components coincident with the optical quasar stronger than $10 \mathrm{mJy}$ (e.g. Owen and Puschell, 1984). This statement usually refers to flux densities of the cores at $5 \mathrm{GHz}$, although most of the central components which have been observed at multiple frequencies appear to have flat radio spectral indices. 


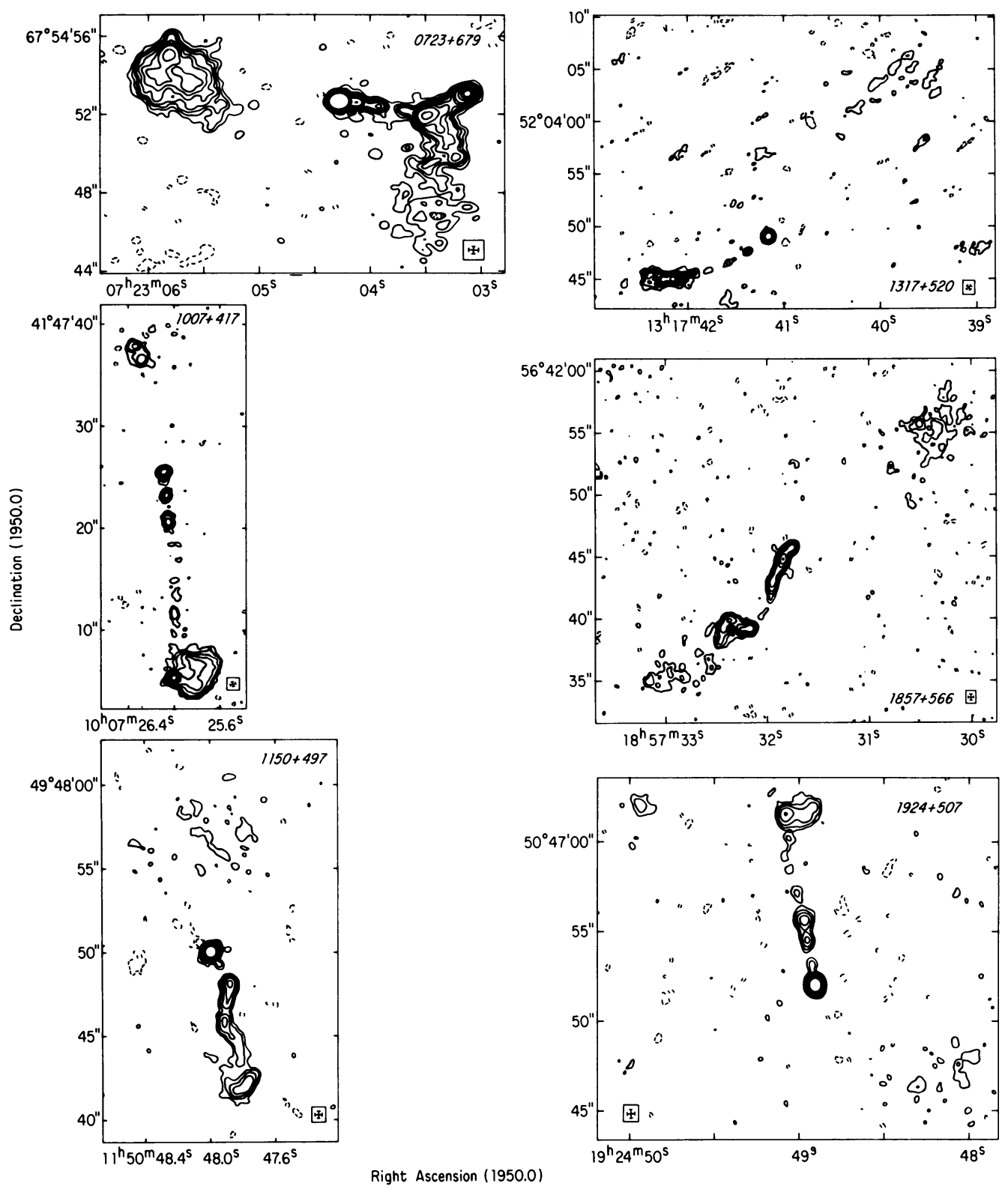

Figure 1. Examples of Radio Quasars with Jets (see Owen and Puschell, 1984). 
Powerful radio galaxies also appear to have jets (usually one-sided) and central components; however, their strength, at least relative to the radio lobes, is weaker. Only a few FRII class radio galaxies (Fanaroff and Riley, 1974) have been observed to possess radio jets (e.g. Perley et al., 1984; Linfield and Perley, 1984) and the detection of these cases has taken long integrations with the VLA and special image processing techniques. Nonetheless when the observations have been made the jets, also one-sided, have been found. Also the central components are weaker. For high redshift radio galaxies with similar or higher luminosities to the quasars mentioned above the central components are almost always weaker than $10 \mathrm{mJy}$, and typically for 3CR sources with redshifts greater than 0.3 are about $1 \mathrm{mJy}$ at $5 \mathrm{GHz}$ (Owen et al. 1981, Owen and Laing, in preparation).

Thus at the $1 \mathrm{Jy}$ level a powerful radio galaxy can usually be distinguished from a quasar by noting the strength of the central component. A strong, one-sided jet is a further tipoff. The structure of the lobes, of course, is a good indicator of the general range of radio luminosity (Fanaroff and Riley, 1974).

\section{Physical Conditions}

The understanding of the physics of the extended structures of quasars is dominated at present by the question of whether or not relativistic motion dominates the observed properties of the systems. This question will be discussed in the next section. Otherwise quasars are usually understood within the context of the beam model which can be studied, although imperfectly, in nearerby radio galaxies where we can observe the radio structure at higher linear resolution and have a better understanding of the external conditions. Nonetheless, a number of physical problems will probably, eventually be best attacked in quasars due to their apparently extreme conditions. Two such problems are 1) the combination of the energy supply and with the bending of quasar jets and 2) the confinement of the emitting structures. Potash and Wardle (1980) discussed the bending and energy supply question for $4 \mathrm{C} 32.69$ and show the apparent contradiction with the standard assumption of the kinetic energy in the flow being converted into radio luminosity. Barthel (1984) has considered this question for high redshift quasars and considered deflections by dense, cool clouds as the origin of the bending. Others (e.g. Gower et al. , 1982 ) have been able to explain some structures by ballistic precession models. However, if the jets act like a fluid as is expected in most modern jet models, a different formalism needs to be used (Hardee ,1984) which has not been applied to many cases. In particular, the quantity, $\omega R / u$ is conserved, where $\omega$ is the wave frequency, $R$ is the jet radius, and $u$ is the jet velocity (Hardee, 1986).

The second major problem is that the minimum pressure implied by the brightness of the extended structure (as high as $10^{-6}$ dynes $-\mathrm{cm}^{-2}$ ) appears to exceed any reasonable external pressure (e.g. Potash and Wardle, 1980; Owen and Puschell, 1984; Barthel, 1984). This situation also appears to be true in more nearby sources such as M87 (Biretta et al, 1983). However, due to their distance, more possible explanations exist for quasars:

1) The pressure may really be higher than we think near some quasars.

2) The jets could be made up of plasmoids in a channel which are each dynamically confined (e.g. Christiansen et al. , 1977).

3) The jets could be magnetically confined as has been suggested for M87. 
4) The jets could simply have a low filling factor (like the broad line region) with high density regions made up of transients locally out of pressure equilibrium (e.g. plasma waves) but with large scale pressure equilibrium maintained. (Borovsky, Taos Jet Meeting 1985). Since $P_{\min } \propto \phi^{3 / 7}$, where $\phi$ is the filling factor, the overall pressure required goes down.

\section{Relativistic Motion, Unified Pictures}

The most important question about the extended structure in quasars is whether or not the observational results can be explained by relativistic motion in the jets. Two general possibilities can be considered:

1) Jets look one-sided due to doppler beaming (e.g. Orr and Browne, 1982).

2) Quasar Jets are intrinsically one-sided (e.g. Wardle and Potash, 1985).

If possibility 1 ) is considered, at least two subclasses of the model can be considered.

a) All quasars are the same and radio quiet quasars are simply beamed away from us (Scheuer and Readhead, 1979).

b) Radio quasars are radio galaxies with their jets pointed close to our line of sight. The cases with jets near the plane of the sky are called radio galaxies and the nuclei in other wavelength bands (e.g. optical) are hidden from us by absorption (Laing, Gull, private communication).

Case 2), intrinsically one-sided jets, also has at least two subclasses.

a) The jets flip-flop back and forth between the two radio lobes (e.g. Rudnick and Edgar, 1984).

b) The jet on one side is invisible either because it simply has fewer losses or because its energy is carried by different particles (e.g. electrons on one-side, protons on the other).

To attack this problem we have three equations describing the relativistic motion which need to be statisically fit by the data.

For $\beta=v / c$ and $\theta=$ the angle between the jet and the line of sight,

1) The intensity of a continuous jet is enhanced by relativistic motion by

$$
S_{o b s}=S_{0} \times(\gamma(1-\beta \cos \theta))^{-(2+\alpha)}
$$

where $S \propto \nu^{-\alpha}$.

For small $\theta$,

$$
S_{o b s} \sim \gamma^{2+\alpha} S_{0}
$$


2) The value of $\beta$ from the ratio, $R$, between the intensity of the approaching and receding sides of a continuous, equal, two-sided jet is

$$
\beta=\frac{R^{\delta}-1}{\cos \theta\left(R^{\delta}+1\right)}
$$

where $\delta=1 /(2+\alpha)$.

3) The apparent superluminal motion of a source is given by

$$
v_{a p p}=\left(v_{j} \sin \theta\right) /(1-\beta \cos \theta)
$$

For $\theta \sim 1 / \gamma, v_{a p p} \sim \gamma v_{j}$.

These three relationships assume isotropic radiation in the frame of the jet and cand be modified somewhat by including a more realistic model (e.g. radiation from shocks, Lind and Blandford, 1985). Relationship 1) must be satisfied statisically given the detailed assumptions of a model. Relation 2) for relativisic motion sets a limit on $\theta$ which must also agree statistically with the model. Relation 3) gives the allowed distribution of $\gamma$ and $\theta$. Sorting out these relationships for any model requires complete data sets and detailed arcsecond and milliacrsecond observations. Such information is not yet available for any large sample. However, some clues are starting to appear as to the answer. (Also see Bridle and Perley for a more lengthy discussion of the observational constraints on this subject.)

Rudnick et al. (1984) have shown that for a few radio quiet (or more correctly radio weak) quasars, the radio emission is extended and resembles observations of nearerby Seyferts. Recent work has also shown that most radio quasars with strong cores possess quite extended radio structure. These sources resemble generally the structure and extended luminosity of radio quasars and galaxies with weaker cores (Browne et al., 1982; Schilizzi and DeBruyn, 1983; Murphy, Browne and Perley, in preparation). These two pieces of information seems to suggest two classes of parent objects with radio quiet quasars resembling active spiral galaxies and radio loud quasars resembles radio active ellipitical galaxies. Thus case 1a) seems unlikely.

Second, the number of distant radio galaxies and the number of quasars in the 3CR are roughly equal. This implies that under $1 \mathrm{~b}$ ) many of the quasars must be as much as 60 degrees from the line of sight.

Third, some correlations exist for and against the beaming hypothesis. Strong cores correlate with one-sided jet structures (Saikia, 1984; Owen and Puschell, 1984). But exceptions exist such as $1857+566$ which has a strong, very distorted jet and a very weak central component (Saikia et al., 1983; Owen and Puschell, 1984). The orientation of the position angle of the core polarization seems to be generally consistent with the expectations of the amplification of small intrinsic misalignments by projection effects near the line of sight ( Saikia and Shastri, 1984). However, the projected linear size distributions for sources with and without jets are not significantly different (Saikia, 1984). 
Fourth, many several quasars or radio galaxies with observed superluminal motion have been found to have large, extended radio structures, often approaching the size of the largest known radio quasars and typical large radio galaxies (e.g. Schilizzi and deBruyn, 1983; Walker, in preparation for 3C120). Also some of the quasars at the upper end of the largest angular size - redshift diagram are being found to have strong central components and one-sided radio jets (Neff and Brown, 1984; Barthel, in preparation; Hintzen and Owen, in preparation).

With these results and others in this volume it is probably still too early to decide the place of relativistic beaming in radio quasars. More careful statistical studies of quasars currently underway have a good chance of settling the question or at least ruling out some models. The next five years should produce very interesting results.

\section{References}

Barthel, P. D. (1984). Ph. D. Thesis, Leiden University

Bridle, A. H. and Perley, R. A. (1984). Ann. Rev. Astron. Astrophys., 22, 319.

Biretta, J. A., Owen, F. N., and Hardee, P. E. (1983). Ap. J. (Letters), 274, L27.

Browne, I. A. W., Clark, R. R., Moore, P. K., Muxlow, T. W. B., Wilkinson, P. N., Cohen, M. H., and Porcas, R. W. (1982). Nature, 299, 788.

Christiansen, W. A., Pacholczyk, A. G., and Scott, J. S. (1977). Nature , 266, 593.

Fanaroff, B. L., and Riley, J. M., (1974). M.N.R.A.S., 101, 31p.

Gower, A. C., Gregory, P. C., Hutchings, J. B., and Unruh, W. G. (1982). Ap. J., $\underline{262}, 478$.

Hardee, P. E. (1984). Ap. J., 287, 523.

Hardee, P. E. (1986). submitted

Lind, K. R., and Blandford, R. D. (1985). Ap. J., 295, 358.

Linfield, R., and Perley, R. A. (1984). Ap. J., 279, 60.

Neff, S. G., and Brown, R. L. (1984). A. J., 195, 195.

Orr, M. J. L. and Browne, I. W. A. (1982). M.N.R.A.S., 200, 1067

Owen, F. N., and Puschell, J. J. (1984). A. J., 89, 932.

Owen, F. N., Puschell, J. J., and Laing, R. A. (1981). I.A.U. Symposium No. 97 , 435.

Perley, R. A., Dreher, J. W., and Cowan, J. J. (1984). Ap. J. (Letters), 285, L35.

Potash, R. I., and Wardle, J. F. C. (1980). Ap. J., 239, 42.

Rudnick, L. , and Edgar, B. K. (1984). Ap. J., $279,74$.

Rudnick, L. , Sitko, M. L. , and Stein, W. A. (1984). A. J., 모, 753.

Saikia, D. J. (1984). M.N.R.A.S., 208, 231.

Saikia, D. J. and Shastri, P. (1984). M.N.R.A.S., 211, 47.

Saikia, D. J., Shastri, P., Cornwell, T. J., and Banhatti, D. G. (1983). M.N.R.A.S., $\underline{203}, 53 P$.

Scheuer, P. A. G., and Readhead, A. C. S. (1979). Nature , 277, 182.

Schilizzi, R. T. , and deBiuyn, A. G. (1983). Nature, 303, 26.

Wardle, J. F. C. and Potash, R. I. (1985). Physics of Energy Transport in Extragalactic Radio Sources, ed. Bridle and Eilek , (Green Bank, NRAO), p30. 


\section{DISCUSSION}

Fabbiano : You mentioned that the difference between core component of quasars and radio galaxies might be explained with beaming or column densities. By looking at FRII 3 CR radio galaxies and quasars we find two different classes of quasars. The double lobed $3 \mathrm{CR}$ quasars appear to be very similar (but brighter than $3 \mathrm{CR}$ galaxies when their core $5 \mathrm{GHz}$ radio luminosities and their X-ray luminosities are compared. They follow the same correlation as the galaxies, suggesting that their cores are only more luminous specimens from radio galaxies cores. A substantial column density in radio galaxies (but not in quasars) would affect this correlation. The radio flat quasars are instead over luminous in radio, for a given $X$-ray luminosity. Beaming or an extra radio component might be present in these objects. 
"Until further notice I offer a free beer for every new QSR having $z>2.5$ ana a steep $(\mathrm{cm}-\mathrm{m})$ radio spectrum."

\footnotetext{
- Peter Barthel (p.182)
} 